\title{
Calcaneus osteomyelitis secondary to Guthrie test. Case report
}

Tugce Tural Kara, M.D. ${ }^{a}$, Tugba Erat, M.D. ${ }^{a}$, Halil Ozdemir, M.D. ${ }^{a}$, Aysun Yahsi, M.D. ${ }^{a}$, Suat Fitoz, M.D. ${ }^{b}$, Ergin Ciftci, M.D. ${ }^{a}$ and Erdal Ince, M.D. ${ }^{a}$

\begin{abstract}
Calcaneus osteomyelitis is a rare infection in newborns. Invasive procedures, prematurity and low birth wight are some causative factors. The clinical signs may be mild. We report a neonate with calcaneous osteomyelitis which was secondary to a Guthrie test sample. She was admitted to hospital with swelling, redness and increased temperature on her heel. Superficial tissue ultrasound showed fluid collection with heavy content was observed on the posterior of the right foot. Puncture and drainage was performed and methicillin-susceptible Staphylococcus aureus was isolated from the drainage culture. Extremity magnetic resonance imaging showed tissue defect in right foot, edema-inflammation and contrast enhancement. Intravenous sulbactam-ampicillin and oral amoxicillin and clavulanic acid were given for 6 weeks. In follow-up, clinical symptoms recovered without curettage. In conclusion; calcaneous osteomyelitis should be considered in the differential diagnosis of swelling on heel in neonates who have been applied to invasive procedures as Guthrie test sample. Key words: calcaneus, screening test, osteomyelitis, neonate.
\end{abstract}

http:/ /dx.doi.org/10.5546/aap.2016.eng.e260

\section{INTRODUCTION}

Acute hematogenous osteomyelitis is a common problem in children that is typically seen in long tubular bones. Calcaneus osteomyelitis is a rare infection in childhood and it may develop by hematogenous spread or direct inoculation such as invasive procedures. ${ }^{1}$ Nowadays, in many hospitals, especially neonatal intensive care units, the heel puncture is used to get blood sample. Although heel puncture is an invasive procedure in neonates, it should not have a

a. Department of Pediatric Infectious Diseases.

b. Department of Pediatric Radiology. Ankara University Medical School, Ankara, Turkey.

E-mail address:

Tugce Tural Kara, M.D.: tugcetural@hotmail.com

Funding: None.

Conflict of interest: None.

Received: 12-7-2015

Accepted: 1-7-2016 higher risk of osteomyelitis if it is made with the right technique. This includes the asepsis and the choice of appropriate site for puncture. Diagnosis may be difficult because clinical symptoms are sometimes mild. ${ }^{2}$ Few cases with calcaneus osteomyelitis have been reported. We present a neonate with calcaneus osteomyelitis which was secondary to Guthrie test sample.

\section{CASE REPORT}

A term female infant weighing $3.6 \mathrm{~kg}$ was born by vaginal delivery without postnatal problems. Guthrie test was done with nozzle on the $7^{\text {th }}$ postnatal day. Three days later, her heel became swelling. In addition, redness and increased temperature also occurred on the puncture site, but fever was not noticed. The patient was admitted to another hospital and she was diagnosed as a hematoma on the heel. The patient did not receive any treatment from the first consultation on the $10^{\text {th }}$ day of life until admission to our hospital at 28 days of life. She was admitted to our hospital because of the increased swelling and redness on the heel.

On physical examination, there was no any pathological finding, except $5 \times 5 \mathrm{~cm}$ swelling, redness, tenderness and increased temperature on the right heel. Patient laboratory test results were: haemoglobin: $15.8 \mathrm{~g} / \mathrm{dl}$, leukocyte count: $16600 / \mathrm{mm}^{3}$ (neutrophils $46 \%$, lymphocytes $50 \%$ and monocytes $4 \%$ ), platelet count: $336000 / \mathrm{mm}^{3}$ and C-reactive protein: 51 $\mathrm{mg} / \mathrm{L}$ and erythrocyte sedimentation rate: $40 \mathrm{~mm} / \mathrm{h}$. Direct X-ray showed osteolytic lesion on the calcaneus which was consistent with osteomyelitis (Figure 1). Superficial tissue ultrasound showed $24 \times 11 \mathrm{~mm}$ fluid collection with heavy content on the posterior of the right foot. Extremity magnetic resonance imaging (MR) showed tissue defect, edemainflammation and contrast enhancement on the right foot (Figure 2A, 2B, 2C). All these findings supported osteomyelitis of the calcaneous bone. Puncture and drainage was performed by pediatric surgery. Methicillin-susceptible Staphylococcus aureus was isolated from the 
drainage culture. Then intravenous sulbactamampicillin treatment was started. In addition abscess drainage was performed two times.

$S$. aureus was detected in nasal swab culture of the patient and her mother. Therefore, decontamination with mupirocin ointment was made for 10 days. Intravenous sulbactamampicillin treatment was given for 3 weeks. In addition oral amoxicillin and clavulanic acid was received for 3 weeks after discharge. At the end of treatment, the heel lesion was completely resolved and acute phase reactants were normal. In follow-up, the patients recovered without complications.

\section{DISCUSSION}

Osteomyelitis is an important infection in childhood. The most affected sites are rapidly growing bones like femur, tibia, humerus that have rich blood supply. Osteomyelitis is caused by the microorganisms which reach to the bone with hematological way or the direct inoculation. ${ }^{1}$ Acute hematogenous osteomyelitis is more common in children. Pathogenic microorganisms come to bone through the nutrient arteries then they pass to the metaphyseal capillaries. The host's immune system is weak in those areas where microorganisms are deposited and accumulated. Bacterial growth moves toward vascular veins and cartilaginous matrix. Vessel walls are obstructed by the proliferation of bacteria and feeding of the bone is blocked. The infection can spread epiphysis, diaphysis and the joint space. This expansion can cause some

FIGURE 1. Lateral radiogaphy of both ankle shows plantar and retrocalcaneal soft tissue swelling and radiolucent area in the posterior portion of the calcaneus bone (arrow).

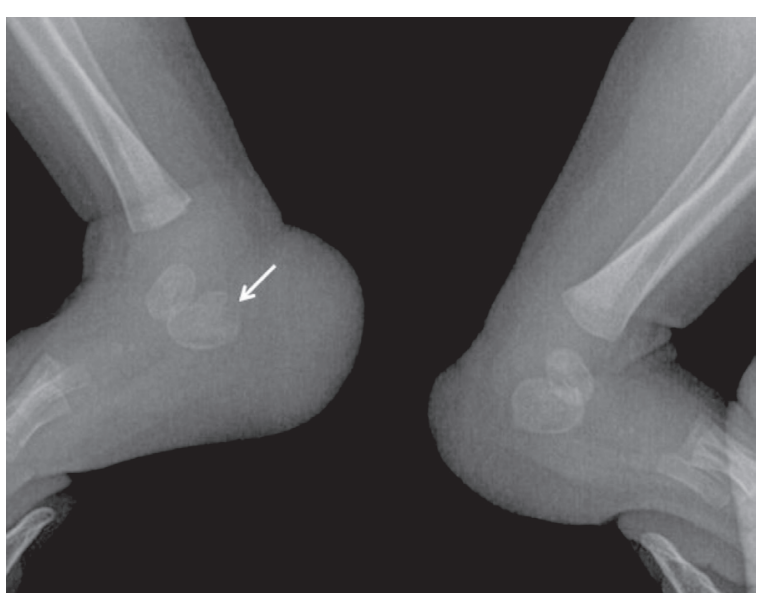

poor results. Growth plate may be damaged and abnormal bone growth may be seen as a complication. ${ }^{3,4}$

FIGURE 2. Sagittal STIR MR image (2A) reveals hyperintense signal in ossification center and cartilage part of calcaneus associated with soft tissue edema. Sagittal contigous T1-weighted contrast-enhanced $M R$ images (2B and 2 C) show strong enhancement in te calcaneal bone and soft tissues.

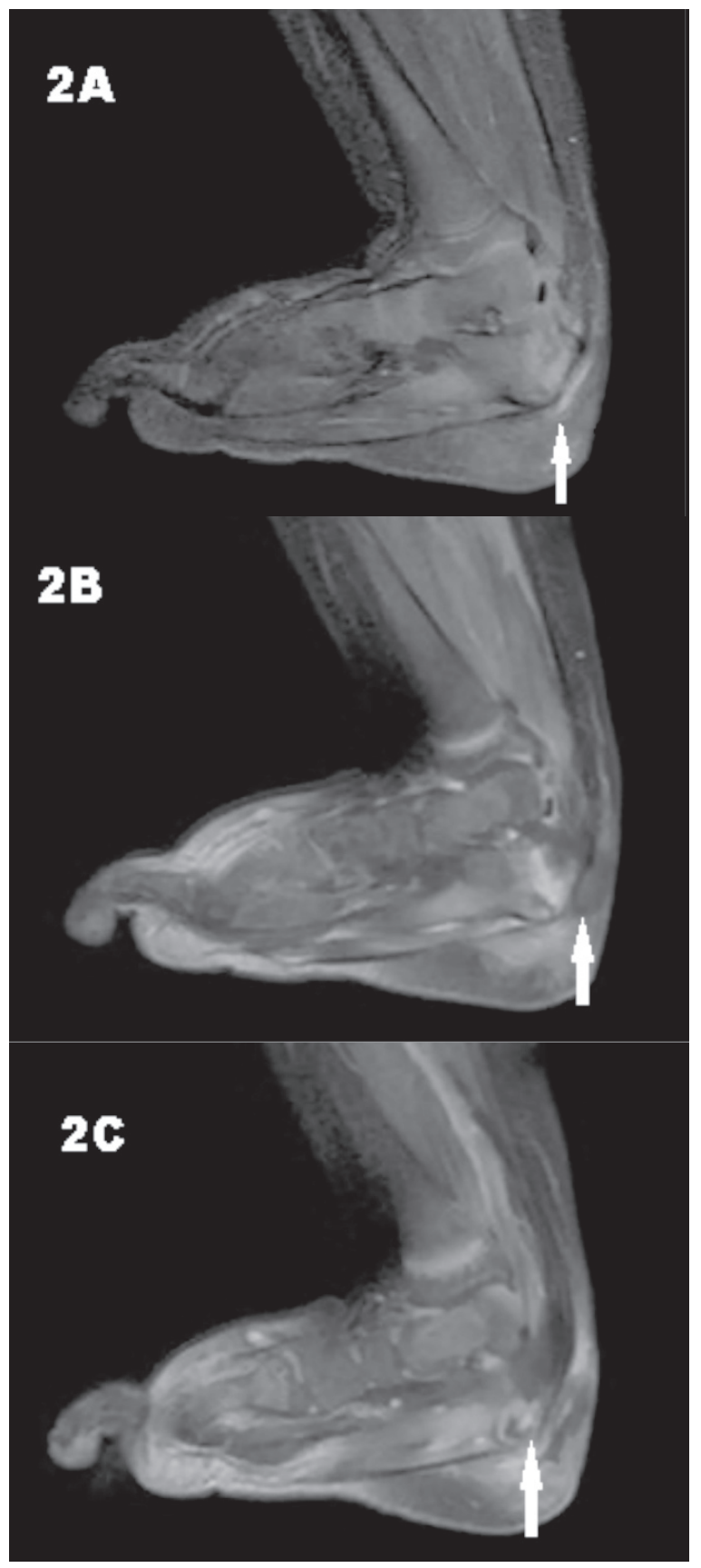


Calcaneus osteomyelitis is an unusual infection in children. Although the incidence of newborn is unknown, it has been frequently reported in the first year of life. It is seen about $3-10 \%$ of all pediatric osteomyelitis. ${ }^{1}$ Invasive procedures, prematurity, low birth wight are some causative factors. ${ }^{5}$ According to the long bone osteomyelitis, diagnosis of calcaneous osteomyelitis may be delayed due to mild clinical symptoms. "Metaphyseal equivalent" area in the neighborhood apophysis is responsible for susceptibility of the calcaneous osteomyelitis. ${ }^{6}$ Calcaneal osteomyelitis may develop with the way which is local spread to bone from infected soft tissue or direct inoculation of the microorganisms through the puncture of the heel. ${ }^{78}$

In literature there are few reports with neonatal calcaneus osteomyelitis. In these cases repeated punctures which were done to get blood sample from hospitalized infants are found to be responsible for osteomyelitis. ${ }^{9-12}$ Only one of them is associated with Guthrie test. In this patient, likewise our patient, osteomyelitis developed after a single puncture and S. aureus was isolated from the drainage culture. ${ }^{13}$

Treatment of calcaneal osteomyelitis is similar to long bone osteomyelitis. Intravenous and oral antibiotics, surgery are used for treatment. However, there is no consensus on the necessary of surgery. ${ }^{14}$ Leigh et al. reported the patients received IV antibiotics for 3 weeks, then oral antibiotics were given for 3 weeks. In addition surgery, aspiration, debridement and washout were performed for needed patients. ${ }^{1}$

Yüksel et al. reported that intravenous cefazolin and gentamicin treatment were given for 4 weeks and curettage was made. ${ }^{13}$ However, our patient received intravenous treatment for 3 weeks. In addition amoxicillin-clavulanate treatment was continueing for 3 weeks. Our patient recovered without currteage. We know that recurrent invasive practices may cause osteomyelitis. However, in our case as well as in that case calcaneous osteomyelitis developed after first Guthrie test. In addition, clinical improvement was seen without curretage which was different from previously reported case. Our case supported sufficient antibiotic therapy was applied, clinical improved will be seen without curettage.

As a result, calcaneous osteomyelitis is an uncommon infection which should be considered in the differential diagnosis of neonates with swelling, redness, and increased in temperature on the heel. We should not forget that invasive procedures may cause osteomyelitis. Therefore aseptic technique_should be performed before the invasive procedures. In the foot, the distance between the skin and the bone is greater in the side surfaces therefore it should avoid puncturing the posterior and central portions. Regarding the puncture depth should not be greater than 2.4 $\mathrm{mm} .{ }^{7}$ Automatic lancets allow better control of penetration depth (near $2.2 \mathrm{~mm}$ ). If calcaneous osteomyelitis is diagnosed, intravenous treatment must be started quickly. Good clinical results will be obtained with adequate therapy.

\section{REFERENCES}

1. Leigh W, Crawford H, Street M, Huang M, et al. Pediatric calcaneal osteomyelitis. J Pediatr Orthop 2010;30(8):888-92.

2. LaMont RL, Anderson PA, Dajani AS, Thirumoorthi MC. Acute hematogenous osteomyelitis in children. J Pediatr Orthop 1987;7(5):579-83.

3. Emslie KR, Nade S. Pathogenesis and treatment of acute hematogenous osteomyelitis: evaluation of current views with reference to an animal model. Rev Infect Dis 1986;8(6):841-9.

4. Mayberry-Carson KJ, Tober-Meyer B,Smith JK, Lambe DW $\mathrm{Jr}$, et al. Bacterial adherence and glycocalyx formation in osteomyelitis experimentally induced with Staphylococcus aureus. Infect Immun 1984;43(3):825-33.

5. Canale ST, Manugian AH. Neonatal osteomyelitis of the os calcis: a complication of repeated heel punctures. Clin Orthop Relat Res 1981;(156):178-82.

6. Nixon GW. Hematogenous osteomyelitis of metaphysealequivalent locations. AJR Am J Roentgenol 1978;130(1):123-9.

7. Blumenfeld TA, Turi GK, Blanc WA. Recommended site and depth of newborn heel skin punctures based on anatomical measurements and histopathology. Lancet 1979;1(8110):230-3.

8. Jaakkola J, Kehl D. Hematogenous calcaneal osteomyelitis in children. J Pediatr Orthop 1999;19(6):699-704.

9. Fernandez-Fanjul JL, Lopez-Sastre J, Coto-Cotallo D, Zazo $\mathrm{C}$, et al. Calcaneus osteomyelitis of newborns caused by diagnostical heel punction (author's transl). Monatsschr Kinderheilkd 1979;127(8):515-8.

10. Waelkens JJ. Osteomyelitis of the calcaneus in newborn infants; who is afraid of a small puncture? Ned Tijdschr Geneeskd 1989;133(33):1641-4.

11. Goldberg I, Shauer L, Klier I, Seelenfreund M. Neonatal osteomyelitis of the calcaneus following a heel pad puncture: a case report. Clin Orthop Relat Res $1981 ;(158): 195-7$.

12. Abril Martin JC, Aguilar Rodriguez L, Albiñana Cilveti J. Flatfoot and calcaneal deformity secondary to osteomyelitis after neonatal heel puncture. J Pediatr Orthop B 1999;8(2):122-4.

13. Yüksel S, Yüksel G, Oncel S, Divanli E. Osteomyelitis of the calcaneus in the newborn: an ongoing complication of Guthrie test. Eur J Pediatr 2007;166(5):503-4.

14. Pääkkönen M, Kallio MJ, Peltola H, Kallio PE. Antibiotic treatment and surgery for acute hematogenous calcaneal osteomyelitis of childhood. J Foot Ankle Surg 2015;54(5):840-3. 